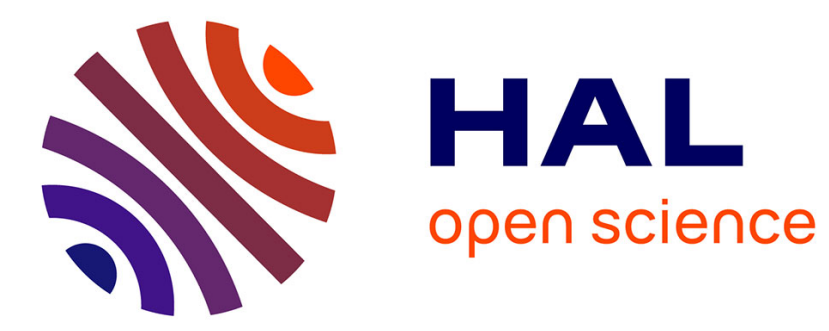

\title{
Double large Barkhausen jump in soft/soft composite microwires
}

G Infante, G A Badini-Confalonieri, R P del Real, M Vázquez

\section{To cite this version:}

G Infante, G A Badini-Confalonieri, R P del Real, M Vázquez. Double large Barkhausen jump in soft/soft composite microwires. Journal of Physics D: Applied Physics, 2010, 43 (34), pp.345002. 10.1088/0022-3727/43/34/345002 . hal-00569682

\section{HAL Id: hal-00569682 \\ https://hal.science/hal-00569682}

Submitted on 25 Feb 2011

HAL is a multi-disciplinary open access archive for the deposit and dissemination of scientific research documents, whether they are published or not. The documents may come from teaching and research institutions in France or abroad, or from public or private research centers.
L'archive ouverte pluridisciplinaire HAL, est destinée au dépôt et à la diffusion de documents scientifiques de niveau recherche, publiés ou non, émanant des établissements d'enseignement et de recherche français ou étrangers, des laboratoires publics ou privés. 


\title{
Double large Barkhausen jump in soft/soft composite microwires
}

\author{
G Infante, G A Badini-Confalonieri, R P del Real and M Vázquez \\ Materials Science Institute of Madrid, CSIC, 28049 Madrid, Spain \\ E-mail: mvazquez@icmm.csic.es
}

\begin{abstract}
The magnetic properties of double layer microwires consisting of a soft FeSiBP amorphous core, an intermediate non-magnetic glass spacer, and a softer FeNi outer shell have been investigated. As in the case of other magnetostatically coupled two-phase systems, the hysteresis loops are characterized by two well-defined Barkhausen jumps corresponding each to the magnetization reversal of the individual phases, separated by a plateau. The strong dipolar interaction that leads to the appearance of the plateau is investigated in terms of the microwire geometry. It is shown that this source of coupling is capable of increasing up to one order of magnitude the switching field of the Fe-rich core. Thus, magnetic bistability can be effectively controlled in this kind of composite wires.
\end{abstract}

PACS: 75.50.Bb, 75.50.Kj, 75.60.Jk, 75.60.Nt, 81.40.Rs

\section{Introduction}

Multilayer systems in the shape of micrometric wires have recently attracted great deal of attention owing to the appearance of interesting magnetic phenomena such as the interaction between magnetic phases via dipolar coupling [1-3]. This effect, together with magnetoelastic interaction, plays the major role on the magnetization process of these composite structures and has proved to optimize most of their properties as the magnetoimpedance [4] and the ferromagnetic resonance [5] responses; both of them useful in sensing applications [6,7].

The dc magnetization reversal in these layered systems has been extensively studied in microwires consisting of a soft core and a hard outer shell in which each phase exhibits completely different magnetization process and consequently distinct hysteresis loops. It has been proved that the lowfield hysteresis curve of the soft core becomes shifted and asymmetric due to the inhomogeneous dipolar field originated by a nearly saturated permanent magnetic shell $[3,8]$, that in addition puts the core under the effect of additional stress [9]. Alternatively, a hard core surrounded by a soft shell has also been considered [10].

In this work, magnetically soft/soft multilayer microwires are proposed; composed of an amorphous bistable FeSiBP core [11] surrounded by an even softer polycrystalline FeNi outer shell. It is shown that dipolar interaction originates two-step hysteresis loops with a plateau, very similar to those observed in other two-phase systems $[12,13]$.
The dependence of the extent of this flat region on the wire dimensions is explained in terms of a simple calculation of the dipolar interaction based on previous models [3] and reveals a peculiar coupling behaviour due to the magnetic configuration here considered. As a consequence, the switching field of the inner Fe-based core can be increased up to ten times its initial value. Therefore, effective control of magnetic bistability is possible in this kind of soft/soft composite wires.

\section{Experimental}

The geometry of the wires under investigation is shown in the SEM images of figure 1. They consist of several concentric layers that were produced as follows: A positive magnetostriction $\left(\lambda_{S} \sim+3 \times 10^{5}\right)$ glass-coated amorphous microwire with nominal composition $\mathrm{Fe}_{76} \mathrm{Si}_{9} \mathrm{~B}_{10} \mathrm{P}_{5}$ was initially obtained by rapid solidification (the $14 \mu \mathrm{m}$ diameter Fe-rich nucleus coated with a $12 \mu \mathrm{m}$ thick Pyrex layer). A nanometric Au layer was sputtered on top of the Pyrex and, finally, a Permalloy shell $\left(\mathrm{Fe}_{20} \mathrm{Ni}_{80}\right)$ with thickness $t$ ranging from 1 to $7.5 \mu \mathrm{m}$ was galvanostatically grown with a current density $j=12$ $\mathrm{mA} / \mathrm{cm}^{2}$ onto the $\mathrm{Au}$ nanolayer in a typical Wattstype solution [14] containing $\mathrm{FeSO}_{4} \cdot 7 \mathrm{H}_{2} \mathrm{O}(8 \mathrm{~g} / \mathrm{l})$, $\mathrm{NiSO}_{4} \cdot 6 \mathrm{H}_{2} \mathrm{O}(125 \mathrm{~g} / \mathrm{l}), \mathrm{NiCl}_{2} \cdot 6 \mathrm{H}_{2} \mathrm{O}(20 \mathrm{~g} / \mathrm{l}), \mathrm{H}_{3} \mathrm{BO}_{3}$ $(40 \mathrm{~g} / \mathrm{l})$, saccharin $(6 \mathrm{~g} / \mathrm{l})$ and purified water. The $\mathrm{pH}$ value was adjusted to 2.8 by adding $\mathrm{KOH}$, the bath was maintained at a temperature of $55^{\circ} \mathrm{C}$ and stirred previously to each plating process. As it can be observed in figure 1, the glass coating provides a smooth substrate so that the FeNi shell grows with reduced roughness. 

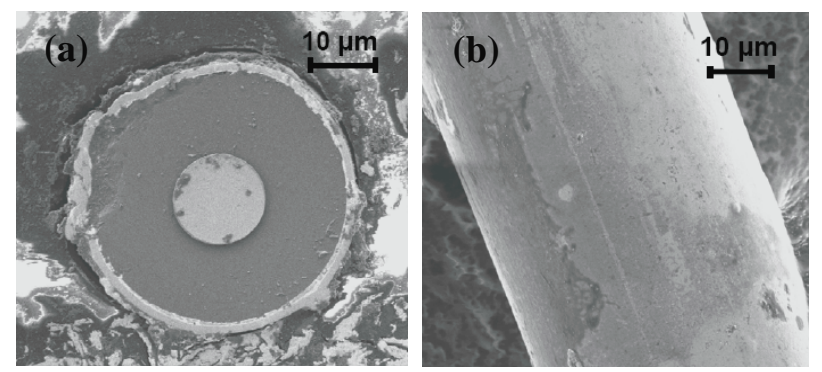

Figure 1. SEM images of an FeSiBP-FeNi microwire with a 2 $\mu \mathrm{m}$ thick outer FeNi shell. (a) cross section and (b) side view showing the outer FeNi layer.

The composition of both magnetic layers was checked by SEM-EDX and found to be homogeneous along the wire radius. The magnetization curves of straight pieces of microwires with length $l$ ranging from 13 to $1 \mathrm{~mm}$ (core and shell have the same $l$ ) were measured in a vibrating sample magnetometer (LOT-Oriel EV7) with the applied field parallel to the wire axis.

\section{Results and discussion}

The hysteresis loops of $13 \mathrm{~mm}$ long FeSiBP-FeNi microwires are presented in figure 2 as a function of the thickness $t$ of the outer FeNi shell. For comparison purpose, the corresponding loop of the bare glass-coated FeSiBP microwire is shown as an inset. As it can be noticed, the magnetization process takes place in two consecutive steps separated by a plateau-like behaviour: the jump below $100 \mathrm{~A} / \mathrm{m}$, which must be ascribed to the reversal of the FeNi shell, and the second jump at higher field (hereafter $H_{\mathrm{sw}}$ ) corresponding to the switching of the inner FeSiBP core. Note that magnetic bistability in the core remains in the whole range of $t$.

It is interesting to analyze how the thickness of the Permalloy coating influences the magnetization reversal process of both magnetic phases (figures 2 and 3): the sharpness of the jump associated to the FeNi shell slightly decreases as its thickness is increased, seemingly due to a change in shape anisotropy. The reversal of the FeSiBP core is also affected: the magnetic volume of the associated large Barkhausen jump keeps constant $\left(6 \times 10^{-3} \mathrm{emu}\right)$ with increasing $t$, while its switching field (i.e. the extent of the plateau) increases continuously from about $150 \mathrm{~A} / \mathrm{m}$ for the thinner shell until reaching a value of $H_{\mathrm{sw}}=577 \mathrm{~A} / \mathrm{m}$ for a $7.5 \mu \mathrm{m}$ thick FeNi shell (figure 2(c)). A strong coupling is thus clearly present between the two magnetic phases.

The switching field of the Fe-rich core is found to increase non-linearly with the thickness of the FeNi shell, as shown in figure 3. Therefore, and since $\lambda_{\mathrm{S}}>0$ for FeSiBP, it should be clarified first if this steep increase is simply due to axial tensile stresses induced by the presence of the FeNi shell. However, this magnetoelastic contribution cannot be easily estimated here since it's not feasible to study the magnetization curve of the inner core in the presence of a nearly demagnetized shell as done in $[4,10]$ for hard magnetic coatings. Despite this, from the observed increase of $H_{\mathrm{sw}}$ in figure 2 with the shell thickness $t$, it can still be inferred that electroplating the shell induces axial tensile stresses in the Fe-rich core. So, in order to simulate their importance on the switching behaviour, $H_{s w}$ was measured as a function of applied tensile stresses $\sigma$ in the bare FeSiBP glass-coated microwire. The results are reported in the inset of figure 3 . According to previous works [15]: $H_{\mathrm{sw}} \propto \sigma^{1 / 2}$ for $\sigma$ larger than the residual stresses of the structure (300-400 $\mathrm{MPa}$ in figure 3; which is an acceptable value for glass-coated microwires [16]). This behavior is explained in terms of the energy required to form the domain wall involved in the magnetization reversal.
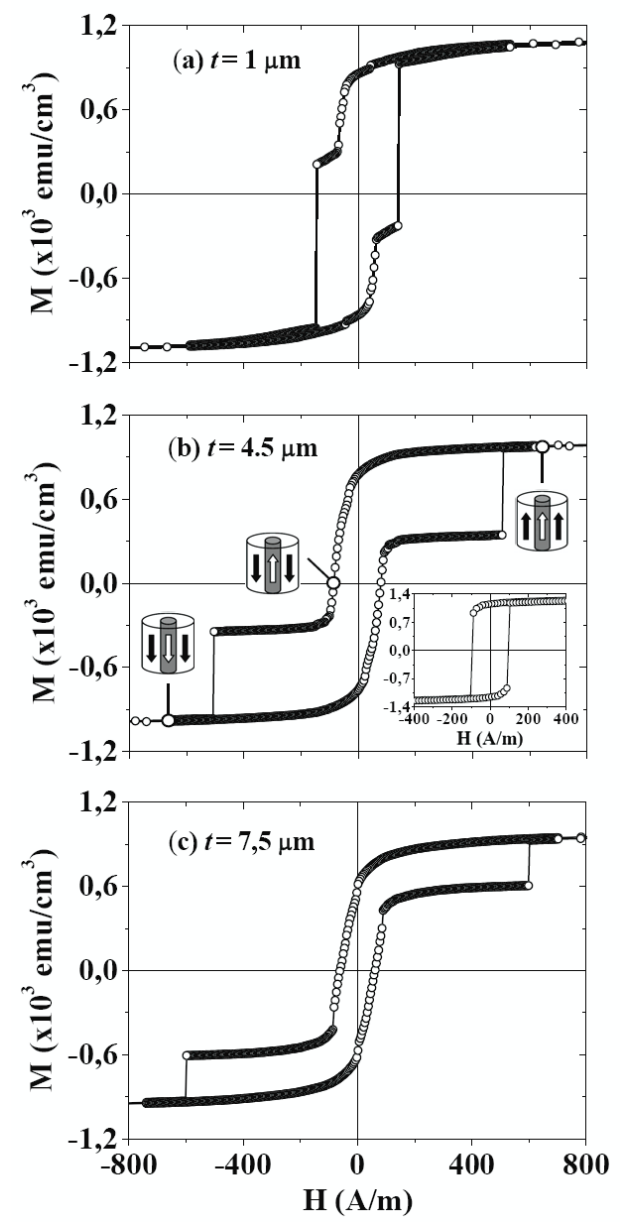

Figure 2. Axial hysteresis loops of FeSiBP-FeNi microwires as a function of the thickness $t$ of the outer FeNi shell: (a) $t=1 \mu \mathrm{m}$, (b) $t=4.5 \mu \mathrm{m}$, (c) $t=7.5 \mu \mathrm{m} . l=13 \mathrm{~mm}$. Inset shows the hysteresis loop of the bare FeSiBP glass-coated microwire. 


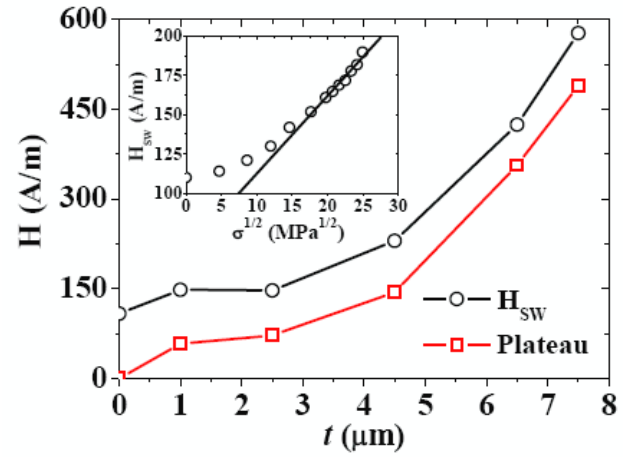

Figure 3. (color online) $H_{s w}$ (black circles) and extent of the plateau (red squares) in FeSiBP-FeNi microwires as a function of the thickness $t$ of the outer FeNi shell. Lines are guidelines for the eye. Inset shows the dependence of $H_{s w}$ on applied tensile stress in the bare FeSiBP microwire. Line represents a linear fit.

Nevertheless, the most important result is that following this saturating trend, unreasonably high stresses $(\sigma \sim 5-10 \mathrm{GPa})$ would be needed to reproduce the large $H_{\mathrm{sw}}$ values found in the composite wires for $t>4.5 \mu \mathrm{m}$. This suggests that magnetoelastic coupling alone cannot explain the observed phenomena, but additional factors should be taken into account. For instance, similar stepped hysteresis loops have been observed for other twophase systems such as a parallel set of bistable ferromagnetic wires [12] or nanocrystalline grains embedded in an amorphous matrix [13]. It has been shown there that the extent of the plateau (i.e. $H_{\mathrm{sw}}$ in the case of long FeSiBP-FeNi microwires) strongly depends on the separation between magnetic phases due to a pure dipolar interaction [12]. Consequently, it seems reasonable to assume that magnetostatic coupling plays the major role in the behavior depicted in figure 3 .

Dipolar coupling controls $H_{\mathrm{sw}}$ in the composite wires as follows: just before the switching of the Fe-rich core, the FeNi shell has already reversed its magnetization and generates a magnetostatic bias field $H_{b}$ in the core that opposes the applied field and therefore delays its $H_{\mathrm{sw}}$, as observed in figure 2. In order to prove this statement, and to justify the results of figure 3 , a calculation of the strength of $H_{b}$, based on previous models for cylindrical geometry [3], has been performed. A simplified but realistic description of the system is adopted: both magnetic phases are saturated just before the switching of the Fe-based core. The magnetostatic potential produced by the outer FeNi microtube is then given by the surface integral:

$$
V(\mathbf{r})=\frac{1}{4 \pi} \int_{S} \frac{\mathbf{n}^{\prime} \cdot \mathbf{M}\left(\mathbf{r}^{\prime}\right)}{\left|\mathbf{r}-\mathbf{r}^{\prime}\right|} d S^{\prime}
$$

Equation (1) has contributions only from the ends of the tube and will be calculated along the Fe-rich core axis $z$, where a simple analytical solution exists. Finally, the dipolar field produced by the FeNi tube in the inner core will be given by:

$$
H_{b}=-\nabla V(\mathbf{r})
$$

As shown in figure 4, thick FeNi shells create a strong demagnetizing $H_{b}$ close to the end of the inner Fe-rich core $(\mathrm{z}<6.5 \mathrm{~mm})$. This is precisely the region where the domain wall depins [11] once $H_{s w}$ is reached. Therefore, the opposing $H_{b}$ field needs to be overcome to switch the core in the composite wires. It should also be noticed that: i) $H_{b}$ takes maximum negative value at nearly $15 \mu \mathrm{m}(\mathrm{z}=6.485$ $\mathrm{mm}$ ) from the end of the core and then decreases towards its centre, and ii) $H_{b}$ changes sign just outside the FeNi shell $(z>6.5 \mathrm{~mm})$. The calculated $H_{b}$ (at $\left.\mathrm{z}=6.485 \mathrm{~mm}\right)$ as a function of the thickness $t$ of the FeNi shell is presented in figure 5. $\mathrm{H}_{b}$ increases nonlinearly with $t$ in qualitative agreement with the experimental results shown in figure 3. The distribution of $H_{b}$ across the core should be considered to obtain better agreement.

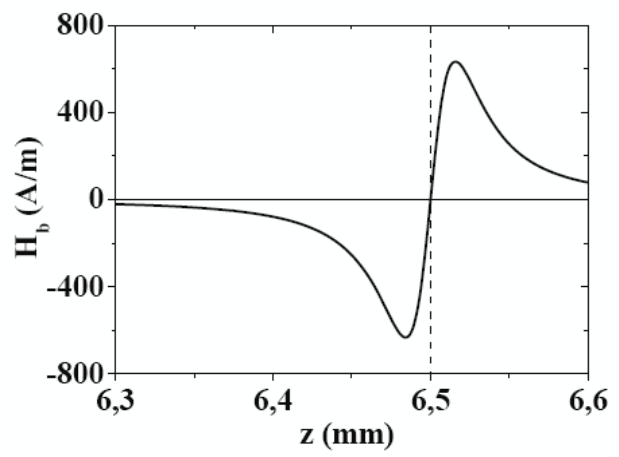

Figure 4. Bias field $H_{b}$ created by a $7.5 \mu \mathrm{m}$ thick FeNi tube along the axis $z$ of the Fe-rich core close to one of its ends (vertical dashed line). $M_{s}=1 \mathrm{~T}$. The tube is of the same length $(13 \mathrm{~mm})$ and inner radius $(19 \mu \mathrm{m})$ to that one in figure 2 .

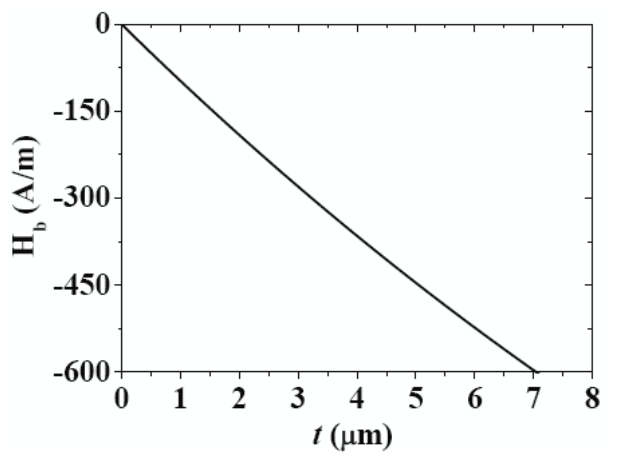

Figure 5. Bias field $H_{b}$ created by an FeNi tube as a function of its thickness $t . H_{b}$ is calculated in one point of the Fe-rich core $15 \mu \mathrm{m}$ from one end. $M_{s}=1 \mathrm{~T}$. The tubes are of the same length $(13 \mathrm{~mm})$ and inner radius $(19 \mu \mathrm{m})$ to those in figure 2 . 
To further analyze the magnetostatic control of $H_{\text {sw }}$ in the FeSiBP-FeNi wires, a study of the influence of the length $l$ of the samples has been performed in a wire with a thick FeNi shell $(t=7.5$ $\mu \mathrm{m})$ where, according to previous discussion, the stronger dipolar effect appears. The results are presented in figure 6 and reveal a stepwise behaviour for the switching field of the Fe-rich core (figure 6(b)): $H_{\text {sw }}$ remains almost constant around $600 \mathrm{~A} / \mathrm{m}$ as $l$ is reduced and suddenly increases more than two times for $l=6 \mathrm{~mm}$. It finally reaches nearly $1300 \mathrm{~A} / \mathrm{m}$ for $l=2 \mathrm{~mm}$. This trend is not observed for the bare glass-coated wire, where $H_{\mathrm{sw}}$ does not depend on $l$, and additionally supports the main role played by the already discussed magnetostatic effect. This result may be explained considering the change in anisotropy studied in FeNi microtubes, with similar dimensions and grown on top of glass fibers without metallic cores, as shown in figure 7 . The length at which the anisotropy in the FeNi tube is observed to change drastically, coincide with the same range of $l$ values where $H_{\mathrm{sw}}$ increases suddenly in the composite wire. The change of easy axis in the FeNi shell when its length is reduced will certainly alter the magnetic pole distribution and consequently modifies the dipolar bias field $H_{b}$ acting in the Ferich core, originating an additional enhancement in its switching field value.
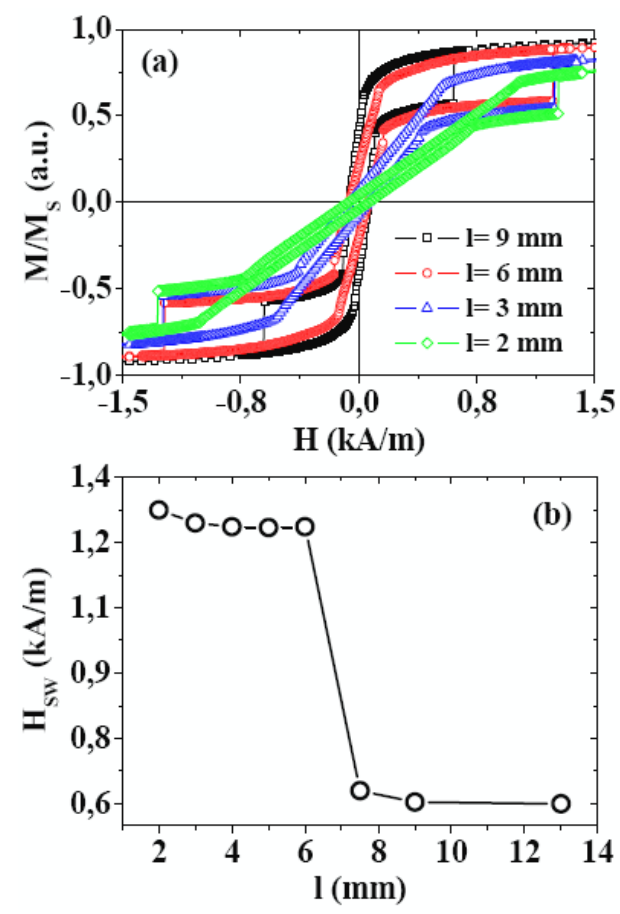

Figure 6. (color online) Influence of the sample length $l$ in an FeSiBP-FeNi microwire with a $7.5 \mu \mathrm{m}$ thick FeNi shell. (a) Axial hysteresis loops. (b) The evolution of $H_{s w}$. Line is a guideline for the eye.

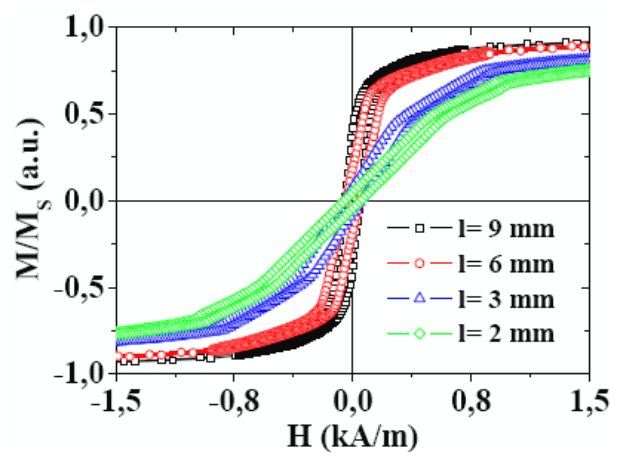

Figure 7. (color online) Axial hysteresis loops a $7.5 \mu \mathrm{m}$ thick FeNi tube as a function of its length $l$. The inner and outer radii are the same ones to those in figure 2 .

It should be noted that reducing the wire length turns into a much complex situation where the simple model here considered, and others based on the same assumptions [3,12], fail to explain the experimental results: for short wires the outer shell is no longer saturated when the core reverses its magnetization (see figure 7) and it is therefore necessary to include the volume contribution in equation (1). This task is out of the scope of the present work.

Finally, we should mention that, according to figure 4, if the FeNi shell is slightly shorter than the Fe-rich core (a few $\mu \mathrm{m}) H_{b}$ is expected to help the core switching. This offers an additional way of controlling its magnetic bistability.

\section{Conclusion}

Bistable magnetic effects have been investigated in soft/soft microwires composed of an FeSiBP core and an FeNi shell. It has been shown that their switching behaviour is very sensitive to any modification in geometry as a consequence of dipolar interaction, which can be effectively controlled within one order of magnitude. The effect of thickness of the outer shell and length of the samples has been analyzed, but other possible methods for fine tuning of bistability in these wires are varying the thickness of the insulating layer or electroplating outer magnetic shells that don't cover the very end of the core.

\section{Acknowledgement}

The authors thank Dr. V. Raposo for valuable discussion. This work was supported by the Spanish Ministry of Science and Innovation (MICINN) project MAT2007-65420-C02-01, G. Infante acknowledges Spanish MICINN FPU fellowships program. 


\section{References}

[1] Torrejón J, Kraus L, Badini-Confalonieri G and Vázquez M 2008 Acta Materialia 56292

[2] Allende S, Escrig J, Altbir D, Salcedo E and Bahiana M 2009 Nanotechnology 20445707

[3] Escrig J, Allende S, Altbir D, Bahiana M, Torrejón J, Badini G and Vázquez M 2009 J. Appl. Phys. 105, 023907

[4] Torrejón J, Vázquez M and Panina L V 2009 J. Appl. Phys. 105033911

[5] Au Y Y and Ingvarsson S 2009 J. Appl. Phys. 106 083906

[6] Mohri K and Honkura Y 2007 Sens. Lett. 5267

[7] Reynet O, Adenot A L, Deprots S, Acher O and Latrach M 2002 Phys. Rev. B 66094412

[8] Rauscher G and Radeloff C 1991 IEEE Trans. Magn. 275238

[9] Torrejón J, Infante G, Merazzo K J and BadiniConfalonieri G A 2008 IEEE Trans. Magn. 443942

[10] Torrejón J, Badini-Confalonieri G A and Vázquez M 2008 J. Appl. Phys. 103 07E712

[11] Infante G, Varga R, Badini-Confalonieri G A and Vázquez M 2009 Appl. Phys. Lett. 95012503

[12] Pereira A, Denardin J C and Escrig J 2009 J. Appl. Phys. 105083903

[13] Lupu N, Óvári T A, Corodeani S and Chiriac H 2008 J. Appl. Phys. 103 07E725

[14] Li X P, Zhao Z J, Seet H L, Heng W M, Oh T B and Lee J Y 2003 J. Appl. Phys. 946655

[15] Aragoneses P, Blanco J M, Dominguez L, González J, Zhukov A and Vázquez M 1998 J. Pys. D: Appl. Phys. 313049

[16] Chiriac H and Óvári T A 1997 Prog. Mater. Sci. 40 333 